\title{
HIGH ENERGY GALACTIC GAMMA RADIATION FROM COSMIC RAYS CONCENTRATED IN SPIRAL ARMS
}

(NASA-TM-X-70818) HIGH ENERGY GALACTIC GAWMA EADIATION FROM COSMIC RAYS CONCENTRATED IN SPIRAL ARMS (NASA) $\$ 3.25$

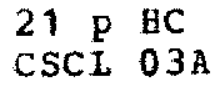

\section{G. F. BIGNAMI}

\author{
C. E. FICHTEL
}

D. A. KNIFFEN

D. J. THOMPSON 


\section{HIGH ENERGY GALACTIC GAMMA RADIATTON}

FROM COSMIC RAYS CONCENTRATED IN SPIRAL ARMS
G. F. Bignami*, C. E. Fichte1, D. A. Kniffen, and D。 J. Thompson NASA/Goddard Space Flight Center
Greenbelt, Maryland 20771

\section{ABSTRACT}

A model for the emission of high energy ( $>100 \mathrm{MeV})$ gamma rays from the galactic disk has been developed and compared to recent SAS-2 observations. In the calculation, it is assumed that (1) the high energy galactic gamma rays result primarily from the interaction of cosmic rays with galactic matter, (2) on the basis of theoretical and experimental arguments the cosmic ray density is proportional to the matter density on the scale of galactic arms, and (3) the matter in the galaxy, atomic and molecular, is distributed in a spiral pattern consistent with density wave theory and the experimental data on the matter distribution that is avai1able, including the 21-cm HI line emission, continuum emission from HII regions, and data currently being used to estimate the $\mathrm{H}_{2}$ density. The calculated $\ell^{\text {II }}$ gamma ray distribution is in good agreement with the SAS-2 observations in both relative shape and absolute flux. As a corollary, the non-uniform cosmic ray distribution of this model tends to support the galactic origin of the fraction of the cosmic rays which are important in the production of high energy photons. Modifications of the basic model show that the gamma ray flux is relatively sensitive to large variations of the assumed distribution of molecular hydrogen in the galaxy.

*NAS-NRC Senior Postdoctoral Resident Research Associate on leave from LFCTR, 'Istituto di Fisica de11' Universita di Milano, Italy 
Subject headings: Galactic gamma rays -- spiral structure -- galactic cosmic rays -- interstellar gas

\section{Introduction}

The most striking feature of the celestial sphere when viewed in the frequency range of high energy gamma rays $(100 \mathrm{MeV})$ is the emission from the galactic plane, which is particularly intense in the galactic longitudinal region from about $300^{\circ}$ to approximately $50^{\circ}$. The energetic galactic gamma rays are generally thought to result primarily from the interaction of cosmic rays with interstellar matter. This concept is supported by the leve1 of intensity being approximately that expected on the basis of cosmic ray interactions with galactic matter (e.g. Kraushaar et al., 1972; Stecker, 1970; Cavallo and Gould, 1971; Kniffen et a1., 1973), and there being no other proposed source which would yield an adequate intensity without what appear to be rather extreme assumptions.

When the problem of the high energy gamma radiation was examined in more detail, Kraushaar et al. (1972) showed that the distribution of cosmic rays appeared inconsistent with a uniform cosmic ray density in the galaxy on the basis of the center to anticenter gamma ray intensity ratio. Considering the geometrical distribution of the intense high-energy gamma radiation, Kniffen et al. (1973) were led to the conclusion that the source of the gamma rays was very likely concentrated in the spiral arm segments. Bignami and Fichtel (1974) proceeded further and proposed that in general the cosmicrays are enhanced where the matter is also greatest, namely in the arm segments. This hypothesis was based on the work of Bierman and Davis (1960) and Parker (1966 and 1969), who showed that the magnetic fields and cosmicrays can only be contained by the mass of the gas through which the magnetic 
fields penetrate; and, hence, they are tied to the matter. The calculated intensity showed general agreement with the longitudinal gamma ray distribution within the limitations of the cylindrical symetry of this simplified mode 1.

There have been many other attempts to explain the high energy galactic gamma radiation, including that of strong et $a 1=(1973)$ and Sch1ickeiser and Thielheim, (1974), who propose correlating the cosmic rays with the current best estimate of the galactic magnetic field, Stecker et al. (1974) who use a central galactic cosmic ray acceleration theory, Cowsik and Voges (1974), who consider the Compton process, and Solomon and Stecker (1974) who assume a constant cosmic ray density, but a high molecular hydrogen density. These theories and a general discussion of previous work are summarized and discussed by Fichtel et a1. (1975).

The specific purpose of this work is to pursue further the encouraging results of the preliminary model of Bignami and Fichtel (1974). Assuming, as in the original paper, that, on the scale of arm segments, the cosmic ray density is proportional to the matter density, the approximation of a cylindrically-symmetric galaxy is replaced by the best available representation of the interstellar gas distribution. The expected high energy gamma ray distribution calculated for this model is compared to recent observations, and the implications of the comparison are discussed.

II. Basic Concept and CaIculation

Beyond the basic theoretical considerations of Parker (1969), mentioned in the introduction which suggest this concept, it is known that the local energy density of the cosmic-rays is about the same as the estimated energy density of the average magnetic fields and the kinetic motion of matter. 
Together the total pressure of these three effects is estimated to be approximately equal to the maximum that the gravitational attraction can hold in equilibrium. This evidence not only supports the theoretical concept of Parker (1969), but also suggests that the'cosmic-ray density may generally be as large as would be expected under quasi-equilibrium conditions. This concept is also given some theoretical support by the calculated slow diffusion rate of cosmic rays (e.g. Parker, 1969; Lee, 1972; Wentze1, 1974) in the magnetic fields of the galaxy based on the cosmic ray lifetime and the small cosmic ray anisotropy, and the likely high production rate of cosmic rays, which together suggest that in general the cosmic rays should be plentiful in a given region and will not move quickly to less dense regions. The concentration of $\mathrm{GeV}$ cosmic rays in spiral arms has also been supported theoretically recently by Wentzel (1974) who uses the basic arguments of Parker (1966 and 1969), but adds a discussion on the importance of cosmic ray scattering to the conclusion. Also, the observed increased thickness of the hydrogen disk at the spiral arms (Jackson and Kellman, 1974) may be attributable to the increased cosmic ray pressure in the arms. Finally, the continuum radio emission generally thought to be synchrotron radiation from cosmic ray electrons interacting with the galactic magnetic fields shows maxima in the direction of the spiral arms (e.g. Price, 1973; Green, 1974), again indicating some concentration of at least the cosmic ray electrons within the spiral arms.

The number and energy spectrum of the gamma rays produced by cosmic rays interacting with interstellar matter have been calculated in detail for the case of the-cosmic radiation in interstellar space by several authors, 
e.g. Stecker (1973) and Cava11o and Gould (1971) for the proton interactions, and Cheung (1974) for the case of cosmic ray electrons using the bremsstrahlung cross sections of Koch and Motz (1959) and the electron spectrum deduced by Daugherty et a1. (1975).

The flux of gamma rays with energies greater than $\mathrm{E}$ ( $\mathrm{E}=100 \mathrm{MeV}$ in this case) at a distance $r$ is given by the expression

$$
\Phi(E)=\left(1 / 4_{\Pi}\right) \int\left[S_{n}(E) g_{n}\left(r, l^{I I}, b^{I I}\right)+S_{e} g_{e}\left(r, \ell^{I I}, b^{I I}\right)\right] N\left(r, \ell^{I I}, b^{I I}\right) d r d \Omega
$$

where $\mathrm{S}$ is the number of gamma rays produced per second on the average for one interstellar hydrogen nucleon plus electron and a cosmic ray density, composition and spectrum equal to that near the earth, $g$ has been introduced to represent the ratio of the cosmic ray density to that in the vicinity of the solar system, the subscripts on $S$ and $g$ refer to cosmic ray nucleons, " $n$ ", and cosmic ray electrons, " $e "$, and $N$ is the total interstellar number density for atomic and molecular hydrogen. The coordinates $r, \ell^{I I}$, and $b^{\text {II }}$ refer to a sun centered coordinate system. For the cosmic ray nucleonic component, the effect of the smaller contribution of nuclei heavier than hydrogen is included in $S$. In the case of the electrons, $S$ includes the appropriately weighted $Z(Z+1)$ factor, which is slightly larger than the 2.0 for atomic hydrogen.

$S_{n}$ is estimated to have a value of $1.3 \cdot 10^{-25} / \mathrm{sec}$ (Stecker, 1973) and $S_{e}$ is $\cdot 2 \cdot 10^{-25} / \mathrm{sec}$ (Cheung, 1974). Considering this fact, it will be assumed in this paper that $S_{n} g_{n}+s_{e} g_{e}$ may be replaced by $S g_{n}$, where $s=s_{n}+s_{e}$, to a good approximation. This approximation neglects the fact that some portion of the electrons seen locally are secondaries (generally estimated to be about $15 \%$ to $40 \%$ Fanselow, et. a1. 1969; Daugherty, et. al., 1975), and the relative 
number of secondaries will vary with the matter density and lifetime. If, however, the secondary electrons represent $25 \%$ of the total local electron population, the secondaries would have to increase by a factor of five to double the total electron component. Since $\mathrm{s}_{e}$ is only $15 \%$ of $\mathrm{s}_{\mathrm{n}}$ above $100 \mathrm{MeV}$, it seems unlikely that this approximation has a serious effect in the high energy region to be considered here. At lower energies electrons are of considerably greater importance, and that problem is the subject of future work. Thus, equation (1) becomes

$$
\Phi(E)=\frac{1}{4 \pi} \cdot 1.5 \cdot 10^{25} \int g_{n}\left(r, l^{I I}, b^{I I}\right) N\left(r, l^{I I}, b^{I I}\right) d r d \Omega
$$

Following the discussion in the introduction, the cosmic ray intensity on the scale of galactic arms is assumed to be proportional to the matter density. Hence, the simplest approach is then to let $g_{n}$ be proportional to the total matter, namely $\mathrm{N}$, and normalize it to the estimated local value; hence:

$$
\mathrm{g}_{\mathrm{n}}\left(\mathrm{r}, \ell^{\mathrm{II}}, \mathrm{b}^{\mathrm{II}}\right)=\mathrm{N}\left(\mathrm{r}, \ell^{\mathrm{II}}, \mathrm{b}^{\mathrm{II}}\right) / \mathrm{N}(\mathrm{r}=0)
$$

\section{Matter Distribution}

\section{A. General}

Although the distribution of the interstellar matter in our galaxy can be studied in a number of ways, the only method that has been able to probe the large scale spiral features over much of the galactic disc is the observation of the $21 \mathrm{~cm}$ line of neutral hydrogen. The radio astronomy measurements can be unfolded and smoothed with the help of the density wave theory to produce a map of the grand design of the spira1 pattern, as in figure 1 , due to Simonson (1975). In what follows, the assumption is made that a11 the interstellar hydrogen, i.e., neutral atomic, ionized atomic and molecular, 
is modulated by the same basic density wave spiral pattern of figure 1. However, the quantitative density distributions for the atomic and the molecular gas will be discussed separately because the measurements so far available on the galactic $\mathrm{H}_{2}$ are characterized by a substantially greater uncertainty than those relating to the atomic component.

B. Atomic Hydrogen

The function $n_{H}\left(r, l^{I I}, b^{I I}\right)$ represents the distribution of the galactic atomic hydrogen densities in atoms per $\mathrm{cm}^{3}$ as a function of the heliocentric distance, $r$, and of the galactic coordinates, $\ell^{I I}$ and $b^{I I}$. In the model to be discussed here, $\mathrm{n}_{\mathrm{H}}\left(\mathrm{r}, \ell^{\mathrm{II}}, \mathrm{b}^{\mathrm{II}}\right)$ is constructed according to the following considerations:

The map in figure 1 gives only the density maxima, or "ridges", of the arms in the equatorial plane. Following the classic Lin-Shu (1967) model as wel1 as simflar assumptions made in more recent work (e.g. Price, 1973; Cowan et. al., 1974), a width of $1 \mathrm{kpc}$ has been given to each arm for simplicity, and the equatorial density has been maintained constant within the arm. The only exception to the $1 \mathrm{kpc}$ width is the local or Orion arm which, following Yuan (1969), has been given a .5 to $.7 \mathrm{kpc}$ width in this mode 1.

The numerical values of the equatorial neutral and ionized atomic : hydrogen $(H I+H I I)$ densities, $n_{H}\left(r, l^{I I}, 0\right)$, were deduced from the radio measurements summarized by Kerr and Westerhout (1965) and Westerhout (1970) using density wave theory. These measurements refer to the $21 \mathrm{~cm}$ Iine for HI and to continuum emission from HII regions. For the region within about $1 \mathrm{kpc}$ from the sun, data are now available both from radio (Falgarone and Lequeux, 1973 and references herein) and recent satellite measurements from 
OAO-2 and Copernicus (Macchetto and Panagia, 1973; Jenkins and Savage, 1974), and the agreement is good giving a value of about .4 atoms/cc. The actual values of $\mathrm{n}_{\mathrm{H}}\left(\mathrm{r}, \ell^{I I}, 0\right)$ which have been used in this work are as follows:

.3 to .2 for interarm regions outside the solar circle

.4 for arms outside the solar circle (e.g. the Perseus arm)

.4 for all interarm regions inside the solar circle and at the sun

.6 for the Orion or local arm

.8 for the Carina arm

1.2 for the Sagittarius arm

1.5 for the other inner arms (Norma, Scutum and $4 \mathrm{kpc}$ ), as we11

as for a central region of $1 \mathrm{kpc}$ radius.

As seen, the arm-interarm density ratio decreases from 3.7 for the inner arms

to 1.5 for the less marked external features. The decrease is supported by the density wave theory (Roberts and Yuan, 1970) and HI measurements of M31 (Guibert, 1974).

The decrease $n_{H}(Z)$ perpendicular to the galactic equatorial plane has been taken to have the quasi-gaussian shape given by Schmidt (1956). The change of this shape. into an exponential one several hundred parsecs above the plane (Cowan et a1.. 1974) introduces only a negligible correction. The variation with galactocentric distance $\widetilde{\omega}$ and galactic longitude of the, half-thickness of the hydrogen disc, $\mathrm{Z} 1 / 2\left(\widetilde{\omega}, \ell^{I I}\right)$, has been taken from the recent measurements of Jackson and Kellman (1974) and varies from 50 pc for $\tilde{\omega} \leq 2 \mathrm{kpc}$ to $400 \mathrm{pc}$ for $\tilde{\omega}>12 \mathrm{kpc}$.

By use of figure 1 and of the information contained in the preceeding discussion, it is then possible to construct a map of $n_{H}(r, \ell I I, 0)$ ia steps of $100 \mathrm{pc}$ in $\mathrm{r}$ and $1^{\circ}$ in $\ell^{\mathrm{II}}$; the third dimension, $\mathrm{b}^{I I}$, is added by 
use of the $n_{H}(Z)$ function with a precision of $1^{\circ}$ in $b^{I I}$ for $r<6$ kpc or $10 \mathrm{pc}$ in $\mathrm{z}$ for $\mathrm{r} \geq 6 \mathrm{kpc}$.

C. Molecular Hydrogen

While the importance of significant amounts of interste1lar $\mathrm{H}_{2}$ for the production of high energy photons has been recognized for some time (Stecher and Stecker, 1970), the measurements presently available on galactic $\mathrm{H}_{2}$ are scarce. They consist of direct measurements in the vicinity of the Sun by the Copernicus Satellite (Spitzer et a1., 1973; Jenkins and Savage, 1974) and of indirect microwave measurements of the line-of-sight densities of $C 0$ molecules for the $\ell^{\text {II }}$ quadrant $0^{\circ}$ to $90^{\circ}$ (Scoville and Solomon, 1974, also given in Solomon and Stecker, 1974). The measurements in the vicinity of the Sun $(\leqslant 1 \mathrm{kpc})$ seem to suggest that $\mathrm{H}_{2}$ is about twice as abundant as the atomic (HI and HII) hydrogen. The interpretation of the 1ine-of-sight $\mathrm{CO}$ emission measurements in terms of the galactic distribution of the $\mathrm{H}_{2}$ density (as given by Solomon and Stecker, 1974) is still at present rather uncertain, both in terms of the unfolding of the co data itself and of its relation to the $\mathrm{H}_{2}$ densities. There is, however, the suggestion that the amount of $\mathrm{H}_{2}$ can be large and variable with the galactocentric distance, $\tilde{\omega}$.

Assuming that the large-scale spiral structure modulates all the interstellar gas, the molecular hydrogen component can be treated as a numerical factor, $\mathrm{K}(\widetilde{\omega})$, times which the atomic density function $\mathrm{n}_{\mathrm{H}}\left(\mathrm{r}, \ell^{\mathrm{II}}, \mathrm{b}^{\mathrm{II}}\right)$ is to be multiplied. In view of the paucity of the experimental evidence, two different cases have been treated. The values of the $k(\tilde{\omega})$ factor are given in Table 1 for both cases. Case 1 assumes that the molecular hydrogen factor, $K(\tilde{\omega})=\mathrm{N}_{\mathrm{H}_{2}}+\mathrm{N}_{\mathrm{HI}}+\mathrm{HII}$ is constant and equa1 to 2 (its value at the $\mathrm{N}_{\mathrm{HI}+\mathrm{HII}}$ 
Sun) up to $\tilde{\omega}=10 \mathrm{kpc}$ and decreases to 1.4 outside the solar circle. Case 2 assumes a $\mathrm{K}(\tilde{\omega})$ dependence that can be deduced from the recent Scoville and Solomon measurements of the CO line-of-sight densities. Using a transformation to a Sun-centered coordinate system, it is then possible to construct the total interstellar density function of eq(1):

$$
\mathrm{N}\left(\mathrm{r}, \ell^{I I}, \mathrm{~b}^{\mathrm{II}}\right)=\mathrm{K}(\widetilde{\mathrm{w}}) \cdot \mathrm{n}_{\mathrm{H}}\left(\mathrm{r}, \ell^{\mathrm{II}}, \mathrm{b}^{\mathrm{II}}\right) \text {. }
$$

The calculation presented here reaches a galactocentric distance $\tilde{w}=16 \mathrm{kpc}$ within which the average equatorial density of the total interstellar gas is $.91 \mathrm{nuclei} / \mathrm{cm}^{3}$.

TABLE 1

Molecular Hydrogen Distribution

$$
\begin{array}{l|l|l|l|l|l|l|l|l|l|l}
\tilde{\omega}(\mathrm{kpc}) & 0 & 2.6 & 4.2 & 5.0 & 6.0 & 7.5 & 8.0 & 10.2 \\
\mathrm{~K}(\tilde{\omega}), \text { Case 1 } & 2 & 2 & 2 & 2 & 2 & 2 & 2 & 1.4 \\
\mathrm{~K}(\tilde{\omega}), \text { Case 2* } & 2_{15} & 1.3 & 2.1 & 3.8 & 2.4 & 2.3 & 1.5 & 1.2
\end{array}
$$

*This distribution is based on microwave observations of Scoville and Solomon (1974) which cover the range $0^{\circ}<l^{I I}<90^{\circ}$, and hence the model calculations are relevant only for that region.

\section{RESULTS AND DISCUSSIONS}

Following the procedure discussed in section II, the galactic gamma ray distribution was calculated using the spiral pattern matter distribution given in section III and shown in figure 1. According to the concept that 
the cosmic ray density was proportional to the matter density, equation 3 was used for $g$. Figure 2 presents the results of the primary calculation using the $K(\tilde{\omega})$ values of Case 1 , Table 1 , along with the recent data of SAS-2 (Fichte1 et a1., 1975) for gamma rays with measured energies greater than $100 \mathrm{MeV}$.

The general features of the longitude distribution are seen to be reproduced we11 by the calculation, particularly considering that the computed results are based directly on the physical model without any norma1ization to the observations. In the central region, the calculation shows the same broad, relatively flat distribution as the data. The peaks corresponding to the Scutum, Norma, and 4-Kpc arms are seen in the longitude ranges $310^{\circ}-320^{\circ}, 330^{\circ}-335^{\circ}$, and $340^{\circ}-345^{\circ}$, respectively, in both the experimenta1 and computed results. On the opposite side of the center, neither the data nor the calculation show any pronounced peaks in the $10^{\circ}-40^{\circ}$ longitude range, while both have a minimum value between $55^{\circ}$ and $65^{\circ}$. Some details of the observed longitude distribution are not reproduced by the model, such as the sma11 excess around $\ell^{I I}=0^{\circ}$ seen in the data and the small peak between $45^{\circ}$ and $50^{\circ}$ in the calculation. The former is understandable since the matter distribution in the center $\left(355^{\circ}<l^{I I}<10^{\circ}\right)$ is very uncertain and probably underestimated especially as far as molecular clouds are concerned. With regard to the latter, the relative1y local Sagittarius $\left(\ell^{\mathrm{II}} \sim 45^{\circ}\right)$ and Cygnus $\left(l^{\mathrm{II}} \sim 80^{\circ}\right)$ peaks are very sensitive to both the local matter concentration and the exact position of the Sun relative to the inner boundary of the Orion Spur. The peak to valley ratio for the Scutum, Norma, and 4 kpc arms (1ongitude ranges of $310^{\circ}-320^{\circ}, 330^{\circ}-335^{\circ}$, and $340^{\circ}$ to $345^{\circ}$ ) seem to be 
more pronounced in the experimental data than in the calculated results. Although the statistical level of the data is such that conclusions of this nature can only be considered tentative, or suggestive, it is true that the simplified approach to the matter density distribution in the arms used here, i.e. a constant value over the width of $1 \mathrm{kpc}$ rather than a rise to a maximum at the ridge from the sides, tends to make the peaks less pronounced than a more realistic model. Both the density wave theory (e.g. Roberts and Yuan, 1970) and measurements of external galaxies (e.g. Mathewson et al., 1972; Guibert, 1974) indicate that the density within an arm decreases outward from the high-compression region of maximum density near the inner side. Before discussing two small variations from this basic model, it should be mentioned that an alternate view of the cosmic ray origin is that the cosmic rays are extragalactic rather than galactic. If they are not galactic then the argument that they must be contained predominantly in the arm segments is no longer valid. In fact, if they represent a uniform extragalactic flux, they would most likely be uniform in the galaxy simply because of the uniform pressure. In this case, a calculation similar to the one just discussed, but with $g$ set equal to 1 in equation (1) gives a ratio for the center region to the anticenter, in regions of no strong sources, of about 2 to 1 rather than the observed value of 7 or 8 to 1 , confirming the earlier similar conclusion mentioned in the introduction. This seems to be a fairly strong piece of evidence against the extragalactic origin of the major fraction of cosmic rays, i.e. of the protons between 1 and $10 \mathrm{GeV}$. It is already known that unless the matter is well below the $10^{-5}$ to $10^{-6}$ atoms $/ \mathrm{cm}^{3}$ range in intergalactic space, the cosmic rays cannot exist throughout the universe at the local density or the diffuse ganma radiation would be well above its measured 
value, although, from that point of view, they could exist at the local level within the local supercluster (e.g. Fichte1, 1974). The evidence here, then, goes a step further and suggests that there is specific evidence to support the local galactic origin for the $\mathrm{GeV}$ cosmic rays observed In the vicinity of the earth, consistent with the presently more common theoretical position.

In the context of this model, it is possible to calculate the total gamma ray output of the galaxy. The value obtained for the galactic gamma ray luminosity $(>100 \mathrm{MeV})$ is $21.0 \cdot 10^{42} \mathrm{sec}^{-1}$.

The first variation to the basic model used the $K(\tilde{\omega})$ distribution given in Case 2, Table 1, based on the work of Scoville and Solomon (1974). Because this molecular hydrogen distribution was derived from measurements in the $0^{\circ}<\ell^{\mathrm{II}}<90^{\circ}$ quadrant, the resulting gamna ray distribution can only be compared with the SAS-2 data in this longitude interval. The calculation shows that whereas the general level in the central region and anticenter regions agree reasonably well with the data, there is a significant peak in the $20^{\circ}-35^{\circ}$ range and an extremely 1arge peak near $\ell^{I I}=0^{\circ}$. These peaks correspond respectively to the "5 kpc" feature and the strong central peak in the co measurements used to derive the molecular hydrogen densities. These peaks stand well above the SAS-2 data, in contrast to the results of the basic model. Without a much more exact knowledge of the molecular hydrogen distribution, it is not possible to discuss the overall comparison of this variation to the basic model. Nevertheless, these results show that the gamma ray flux is relatively sensitive to the distribution of molecular hydrogen. 
In the second variation of the bastc mode1, the possibility was considered that the cosmic rays extend beyond the galactic hydrogen dis. This calculation set the thickness of the cosmic ray distribution at twice the thickness of the hydrogen disk, while still normalizing the cosmic ray flux to the local value. The calculation shows that a thicker cosmic ray disk has little effect on the longitude distribution except to increase its absolute value somewhat, primarily because both matter and cosmic rays are necessary to produce gamma rays, and therefore, extending the width of only one does not create a very marked gamma ray increase. Since the resulting distribution is very similar to Figure 2, with only the intensity being slightly higher everywhere, the results are not shown here.

Both the basic model and the variation in which the cosmic rays are assumed to have a thicker disk than the matter have been used to compute the latitude distribution for the gamma radiation in the range $330^{\circ}<\ell^{\mathrm{II}}<30^{\circ}$. Folding the computed results through the SAS-2 angular resolution function for high energy gamma rays produces a distribution which is wider than the angular resolution itself, but not quite as wide as the experimental latitude distribution (Fichtel, et al., 1975). This small additional broadening may be due to local matter (e.g. Lindblad, et a1., 1973) which is not Included in the present model and does not alter the predicted longitude distribution significantly. 


\section{v. CONCLUSION}

The good agreement of the predicted intensity distribution with the high energy gamma ray data from SAS-2 seems to be a strong argument for the basic thesis of this paper, namely that the cosmic ray density is correlated with the matter density, which on a broad scale is predominately in a spiral arm density wave pattern, and that the observed gamma rays result primarily from cosmic ray interactions with the interstellar matter. As a corollary, this uneven cosmic ray distribution tends to support the galactic origin of the fraction of the cosmic rays which are important in the production of high energy photons.

Finally, the association of the high energy galactic gamma rays with cosmic ray-interstellar matter interactions suggests that, in the future, gamma ray astronomy together with astronomy at other wavelengths holds great promise for the study of the galaxy both in terms of the matter distribution and the cosmic ray pressure distribution. Gamma ray instruments of the future will have the sensitivity and the angular resolution to study the cosmic ray matter distribution not only on the broad scale of arms, but on the finer scale of variations within arms. Then the question of cosmic ray expansion from sources and their propogation and distribution should also be studied in detall to see the effects of the cosmic ray gas pressure and to study galactic structure in finer detail. 


\section{REFERENCES}

Bierman, L。 and Davis, L. 1960, Zs. F. Ap., 51, 19.

Bignami, G. F. and Fichtel, C. E. 1974, Ap. J. Letters, 189, L65.

Caval1o, G. and Gould, R. J。 1971, Nuovo Cim., 2B, 77.

Cheung, C., 1974, Private communication.

Cowan, J. J., Kafatos, Mo, and Rose, W. K. 1974, "Sources of Excitation in the Interstellar Gas and Galactic Structure", U. of Maryland preprint.

Cowsik, R。 and Voges, W. 1974, "Inverse Compton Gamma Rays from the Galactic Disc", ESLAB Symposium on Ganma Ray Astronomy, Frascati (Italy).

Daugherty, J. K。, Hartman, R. C., and Schmidt, P. J. 1975, to be published in Ap. J.

Falgarone, E., and Lequeux, J. 1973, Astron. and Astrophys., 28, 313 . Fanselow, J. L., Hartman, R. C., Hildebrand, R. H., and Meyer, P。 1969, Ap. J. 158, 771.

Fichte1, C. E。, 1974, paper presented at the Royal Society Meeting on the Origin of Cosmic Rays, London, February 1974.

Fichte1, C. E., Hartman, R. C., Kniffen, D. A., Thompson, D. J., Bignami, G. F., Öge1man, H., Öze1, M. E., Tümer, T., 1975, to be pub1ished Ap.J.

Green, A., 1974, Astron. and Astrophys. Supp1. Series 18, 267.

Guibert, J. 1974, Astron. and Astrophys。 30, 353.

Jackson, P. D。 and Kellman, S. A。 1974, A. J. 190, 53. 
Jenkins, E. B. and Savage, B. D., 1974, Ap. J. 187, 243.

Kerr, F. J. and Westerhout, G. 1965, in "Stars and Stellar Systems" $\underline{5}, 167$.

Kniffen, D. A., Hartman, R. C., Thompson, D. J. and Fichte1, C. E. 1973, Ap.J. Letters, 186, L105.

Koch, M. W. and Motz, J. W. 1959, Rev. Mod. Phys., 31, 920.

Kraushaar, W. L., Clark, G. W., Garmire, G. P., Borken, R॰, Highbie, P., Leong, V. and Thorsos, T. 1972, Ap. J., 177, 341.

Lee, M. A. 1972, Ap. J., 178, 837.

Lin, C. C., Shu, F. G. 1967, in "Radio Astronomy and the Galactic System", ed. H. van Woerden, Acad. Press, New York, p. 313.

Lindblad, 0., Grape, K., Sanqvist, A. and Schober, J. 1973, Astron. and Astrophys . 24, 309 .

Macchetto, F. and Panagia, N. 1973, Astron。 and Astrophys. 28, 313.

Mathewson, D. S., van der Kruit, P. C., and Brown, W. N. 1972, Astron. and Astrophys., 17, 468 .

Parker, E. N。 1966, Ap. J., 145, 811.

Parker, E. N. 1969, Space Sci. Rev。, 9, 654 .

Price, R. M. 1974, Astron, and Astrophys. 33, 33.

Roberts, W. W. and Yuan, C. 1970, Ap. J., 161, 887.

Schlickeiser, R. and Thielheim, K. O. 1974, Astron. and Astrophys. 34, 167 .

Schmidt, M., 1956, B.A.N., $\underline{13}, 247$.

Scoville, N. Z, and Solomon, P. M. 1974, to be published in Ap. J. 
Stimonson, S。C.IIT, 1975, CNRS Paris Colloquium, Sept. 1974, L. Weliachew, editor, to be published in 1975.

Solomon, P.M. and Stecker, F。W. 1974, "Radio and Gamma Ray Evidence for a Molecular Arm Feature at 5 kpc from the Galactic Center," ESLAB Symposium on Gamma Ray Asțronomy, Frascati (Italy).

Spitzer, L。, Drake, J. F。, Jenkins, E。 B。, Morton, D。 C。, Rogerson, J。 B., and York, D. B., 1973, Ap. J. Letters, 181, L116。

Stecher, T。P. and Stecker, F. W。, 1970, Nature, 226, 1234.

Stecker, F。W.1970, Ap. and Space Sci。, 6, 377.

Stecker, F。W. 1973, Ap. J., 185, 499 .

Stecker, F。 W。, Puget, J。 L。, Strong, A.W. and Bredekamp, J。H. 1974, Ap. J。 Letters, 188, L59。

Strong, A. Wo, Wdowczyk, Jo and Wolfendale, A. W. 1973, in Gamma Ray Astrophysics (ed. F。W. Stecker \& J。I. Trombka), U. S. Govt. Printing off。, Wash。, 239。

Wentzel, D. G. 1974, Annual Review of Astronomy and Astrophysics, $\underline{12}$, 9. 71 .

Westerhout, Go, 1970, in "Galactic Astronomy", Chin and Murie1 eds., Gordon and Breach, p. 170 .

Yuan, C., 1969, Ap. J。 158, 871. 


\section{FIGURE CAPTIONS}

Figure 1 -- A, smoothed spatial diagram of the locations of the maxima of the galactic matter density deduced from $21 \sim \mathrm{cm}$ HI 1 ine measurements and the density wave theory (Simonson, 1975).

Figure 2 -- Longitude distribution of the galactic high energy gamma ray emission deduced from the spiral arm model, shown by a heavy solid line. The SAS-2 data points, connected by a thin line, are shown for comparison. The increase around $\ell^{I I}=265^{\circ}$ is due to the Vela supernova remnant. The increase from $\ell^{I I}=180^{\circ}$ to $205^{\circ}$ is due to the Crab nebula and possibly other sources. (Fichtel, et al., 1975). Both the data and the calculation are summed from $-10^{\circ}$ to $+10^{\circ}$ in galactic latitude. The diffuse background, shown by a dashed line, has been added to the model calculation to give the gamma ray flux shown by the heavy solid line. 


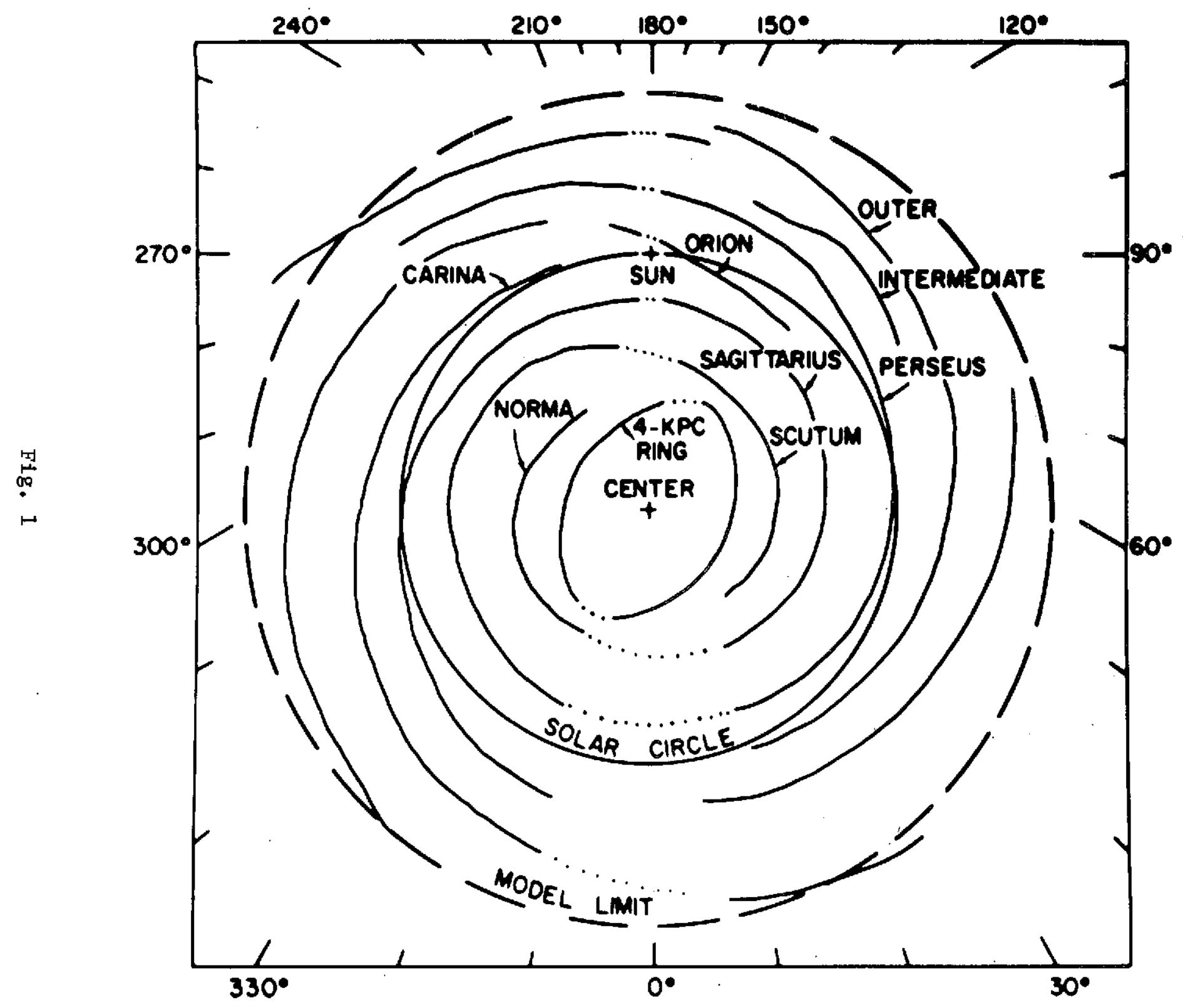




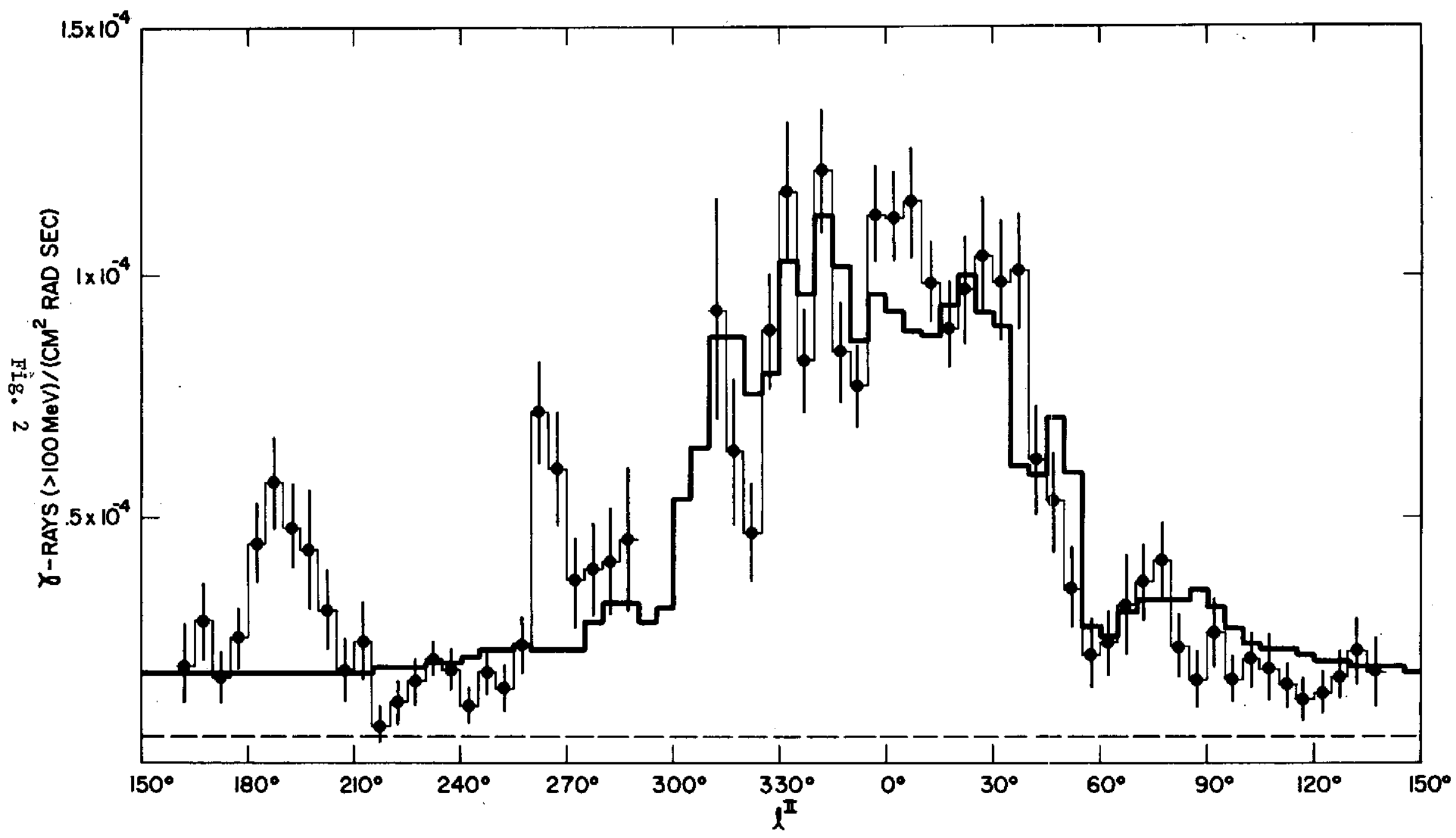

